\title{
SECOND-ORDER COMPUTATIONAL HOMOGENISATION FOR THICK SHELL MODELS: APPLICATION TO NON-LINEAR MULTISCALE ANALYSIS OF COMPOSITES
}

\author{
Aewis K.W. Hii \\ Bristol Composites Institute, University of Bristol \\ *aewis.hii@bristol.ac.uk
}

Advanced composites often have complex material morphology that spans several length scales in the thickness direction, which presents significant challenges for computational analyses. The so-called shell-based computational homogenisation can be used to analyse this class of problems, where it homogenises the nonlinear micro/mesoscopic phenomena using an effective shell model at the macroscale. The homogenisation framework for thin shell models was first proposed by Coenen et al. [1]. Recently, more research efforts have been focused on developing homogenisation techniques for thick shells, to incorporate the effects of through-thickness deformation during scale transition. However, it has been widely reported that classical homogenisation techniques cannot properly represent the macroscopic transverse shear and through-thickness normal strains at the fine scale; and that their homogenised stress resultants are inaccurate when compared to analytical solutions, showing a dependency on the slenderness of the micro/mesomodels [2-4]. As such, the proper treatments for macroscopic throughthickness shell quantities during downscaling remain a prominent research challenge.

The key contribution of this work is the development of a nonlinear second-order computational homogenisation framework for thick shell models. We have proposed a complete set of minimal boundary conditions, such that the correct average membrane, bending, and most importantly the transverse shear and through-thickness normal strains can be obtained during downscaling. In addition, the corresponding fine scale solution yields the correct stress resultants that are convergent and have no size dependency. The framework also allows for kinematic assumptions - such as the inextensibility and the plane stress conditions (ReissnerMindlin), constant thickness strain (6-parameter formulation) and linearly varying thickness strain (7-parameter formulation) - to be enforced at the fine scale. Furthermore, full geometric nonlinearity can be included in both the micro/meso- and macroscopic models, propagating the effects of large displacements and rotations, as well as instability between length scales during an analysis. In a series of benchmarks, the nonlinear multiscale framework is applied to the bending and post-buckling analyses of thick laminates and sandwich panels, where excellent agreements are found between the concurrent multiscale and the full-scale model solutions.

\section{References}

[1] Coenen, E.W.C., Kouznetsova, V.G., \& Geers, M.G.D. (2010). Computational homogenization for heterogeneous thin sheets. International Journal for Numerical Methods in Engineering, 83(8-9), 1180-1205.

[2] Helfen, C., \& Diebels, S. (2013). A numerical homogenisation method for sandwich plates based on a plate theory with thickness change. Journal of Applied Mathematics and Mechanics, 93(2-3), 113-125.

[3] Gruttmann, F., \& Wagner, W. (2013). A coupled two-scale shell model with applications to layered structures. International Journal for Numerical Methods in Engineering, 94(13), 1233-1254.

[4] Främby, J., Brouzoulis, J., \& Fagerström, M. (2016). Assessment of two methods for the accurate prediction of transverse stress distributions in laminates. Composite Structures, 140, 602-611. 